\title{
SCHOTTKY VIA THE PUNCTUAL HILBERT SCHEME
}

\author{
MARTIN G. GULBRANDSEN AND MARTÍ LAHOZ
}

\begin{abstract}
We show that a smooth projective curve of genus $g$ can be reconstructed from its polarized Jacobian $(X, \Theta)$ as a certain locus in the Hilbert scheme $\operatorname{Hilb}^{d}(X)$, for $d=3$ and for $d=g+2$, defined by geometric conditions in terms of the polarization $\Theta$. The result is an application of the Gunning-Welters trisecant criterion and the Castelnuovo-Schottky theorem by Pareschi-Popa and Grushevsky, and its scheme theoretic extension by the authors.
\end{abstract}

\section{INTRODUCTION}

Let $(X, \Theta)$ be an indecomposable principally polarized abelian variety (PPAV) of dimension $g$ over an algebraically closed field $k$ of characteristic different from 2 . The polarization $\Theta$ is considered as a divisor class under algebraic equivalence, but for notational convenience, we shall fix a representative $\Theta \subset X .(X, \Theta)$ being indecomposable means that $\Theta$ is irreducible.

The geometric Schottky problem asks for geometric conditions on $(X, \Theta)$ which determine whether it is isomorphic, as a PPAV, to the Jacobian of a nonsingular genus $g$ curve $C$. The Torelli theorem then guarantees the uniqueness of the curve $C$ up to isomorphism. One may ask for a constructive version: can you "write down" the curve $C$, starting from $(X, \Theta)$ ? Even though one may embed $C$ in its Jacobian $X$, there is no canonical choice of such an embedding, so one cannot reconstruct $C$ as a curve in $X$ without making some choices along the way. We refer to Mumford's classic [11] for various approaches and answers to the Schottky and Torelli problems, and also to Arbarello [1], Beauville [2] and Debarre [3] for more recent results.

In this note, we show that any curve $C$ sits naturally inside the punctual Hilbert scheme of its Jacobian $X$. We give two versions: firstly, using the Gunning-Welters criterion [7, 14], characterizing Jacobians by having many trisecants, we reconstruct $C$ as a locus in $\operatorname{Hilb}^{3}(X)$. Secondly, using the Castelnuovo-Schottky theorem, quoted below, we reconstruct $C$ as a locus in $\operatorname{Hilb}^{g+2}(X)$. In fact, for any indecomposable PPAV $(X, \Theta)$, we define a certain locus in the Hilbert scheme $\operatorname{Hilb}^{d}(X)$ for $d \geq 3$, and show that this locus is either empty, or one or two copies of a curve $C$, according to whether $(X, \Theta)$ is not a Jacobian, or the Jacobian of the hyperelliptic or nonhyperelliptic curve $C$. Then we characterize the locus in question for $d=3$ in terms of trisecants, and for $d=g+2$ in terms of being in special position with respect to $2 \Theta$-translates.

2010 Mathematics Subject Classification. Primary 14H42; Secondary 14C05.

Both authors are partially supported through the Research Council of Norway grant Sheaves on abelian varieties, grant number 230986. M.L. is partially supported by MTM2012-38122-C03-02. 
To state the results precisely, we introduce some notation. For any subscheme $V \subset X$, we shall write $V_{x} \subset X$ for the translate $V-x$ by $x \in X$. Let $\psi: X \rightarrow \mathbb{P}^{2^{g}-1}$ be the (Kummer) map given by the linear system $|2 \Theta|$.

Theorem A. Let $Y \subset \operatorname{Hilb}^{3}(X)$ be the subset consisting of all subschemes $\Gamma \subset X$ with support $\{0\}$, with the property that

$$
\left\{x \in X \mid \Gamma_{x} \subset \psi^{-1}(\ell) \text { for some line } \ell \subset \mathbb{P}^{2^{g}-1}\right\}
$$

has positive dimension. Then $Y$ is closed and

(1) if $X$ is not a Jacobian, then $Y=\emptyset$;

(2) if $X \cong \operatorname{Jac}(C)$ for a hyperelliptic curve $C$, then $Y$ is isomorphic to the curve $C$;

(3) if $X \cong \operatorname{Jac}(C)$ for a non-hyperelliptic curve $C$, then $Y$ is isomorphic to a disjoint union of two copies of $C$.

The proof is by reduction to the Gunning-Welters criterion; more precisely to the characterization of Jacobians by inflectional trisecants. Note that the criterion defining $Y$ only depends on the algebraic equivalence class of $\Theta$, and not the chosen divisor.

For the second version, we need some further terminology from [12] and [6].

Definition 1.1. A finite subscheme $\Gamma \subset X$ of degree at least $g+1$ is theta-general if, for all subschemes $\Gamma_{d} \subset \Gamma_{d+1}$ in $\Gamma$ of degree $d$ and $d+1$ respectively, with $d \leq g$, there exists $x \in X$ such that the translate $\Theta_{x}$ contains $\Gamma_{d}$, but not $\Gamma_{d+1}$.

Definition 1.2. A finite subscheme $\Gamma \subset X$ is in special position with respect to $2 \Theta$-translates if the codimension of $H^{0}\left(X, \mathscr{I}_{\Gamma}\left(2 \Theta_{x}\right)\right)$ in $H^{0}\left(\mathscr{O}_{X}\left(2 \Theta_{x}\right)\right)$ is smaller than $\operatorname{deg} \Gamma$ for all $x \in X$.

Again note that these conditions depend only on the algebraic equivalence class of $\Theta$. The term "special position" makes most sense for $\Gamma$ of small degree, at least not exceeding $\operatorname{dim} H^{0}\left(\mathscr{O}_{X}\left(2 \Theta_{x}\right)\right)=$ $2^{g}$.

Our second version reads:

Theorem B. Let $Y \subset \operatorname{Hilb}^{g+2}(X)$ be the subset consisting of all subschemes $\Gamma \subset X$ with support $\{0\}$, which are theta-general and in special position with respect to $2 \Theta$-translates. Then $Y$ is locally closed, and

(1) if $X$ is not a Jacobian, then $Y=\emptyset$;

(2) if $X \cong \operatorname{Jac}(C)$ for a hyperelliptic curve $C$, then $Y$ is isomorphic to the curve $C$ minus its Weierstraß points;

(3) if $X \cong \operatorname{Jac}(C)$ for a non-hyperelliptic curve $C$, then $Y$ is isomorphic to a disjoint union of two copies of $C$ minus its Weierstraß points.

The proof of Theorem B is by reduction to the Castelnuovo-Schottky theorem, which is the following: 
Theorem 1.3. Let $\Gamma \subset X$ be a finite subscheme of degree $g+2$, in special position with respect to $2 \Theta$-translates, but theta-general. Then there exist a nonsingular curve $C$ and an isomorphism $\operatorname{Jac}(C) \cong X$ of $\operatorname{PPAV} s$, such that $\Gamma$ is contained in the image of $C$ under an Abel-Jacobi embedding.

Here, an Abel-Jacobi embedding means a map $C \rightarrow \operatorname{Jac}(C)$ of the form $p \mapsto p-p_{0}$ for some chosen base point $p_{0} \in C$. This theorem, for reduced $\Gamma$, is due to Pareschi-Popa [12] and, under a different genericity hypothesis, Grushevsky $[4,5]$. The scheme theoretic extension stated above is by the authors [6]. The scheme theoretic generality is clearly essential for the application in Theorem B.

We point out that the Gunning-Welters criterion is again the fundamental result that underpins Theorem 1.3, and thus Theorem B. More recently, Krichever [9] showed that Jacobians are in fact characterized by the presence of a single trisecant (as opposed to a positive dimensional family of translations), but we are not making use of this result.

\section{Subschemes of Abel-Jacobi Curves}

For each integer $d \geq 1$, let

$$
Y_{d} \subset \operatorname{Hilb}^{d}(X)
$$

be the closed subset consisting of all degree $d$ subschemes $\Gamma \subset X$ such that

(i) the support of $\Gamma$ is the origin $0 \in X$,

(ii) there exists a smooth curve $C \subset X$ containing $\Gamma$, such that the induced map $\operatorname{Jac}(C) \rightarrow X$ is an isomorphism of PPAV's.

We give $Y_{d}$ the induced reduced scheme structure.

We shall now prove analogues of (1), (2) and (3) in Theorems A and B for $Y_{d}$ with $d \geq 3$ :

Proposition 2.1. With $Y_{d} \subset \operatorname{Hilb}^{d}(X)$ as defined above, we have:

(1) If $X$ is not a Jacobian, then $Y_{d}=\emptyset$.

(2) If $X \cong \operatorname{Jac}(C)$ for a hyperelliptic curve $C$, then $Y_{d}$ is isomorphic to the curve $C$.

(3) If $X \cong \operatorname{Jac}(C)$ for a non-hyperelliptic curve $C$, then $Y_{d}$ is isomorphic to a disjoint union of two copies of $C$.

As preparation for the proof, consider a Jacobian $X=\operatorname{Jac}(C)$ for some smooth curve $C$ of genus $g$. It is convenient to fix an Abel-Jacobi embedding $C \hookrightarrow X$; any other curve $C^{\prime} \subset X$ for which $\operatorname{Jac}\left(C^{\prime}\right) \rightarrow X$ is an isomorphism is of the form $\pm C_{x}$ for some $x \in X$. Such a curve $\pm C_{x}$ contains the origin $0 \in X$ if and only if $x \in C$. Hence $Y_{d}$ is the image of the map

$$
\phi=\phi_{+} \coprod \phi_{-}: C \coprod C \rightarrow \operatorname{Hilb}^{d}(X)
$$

that sends $x \in C$ to the unique degree $d$ subscheme $\Gamma \subset \pm C_{x}$ supported at 0 , with the positive sign on the first copy of $C$ and the negative sign on the second copy. 
More precisely, $\phi$ is defined as a morphism of schemes as follows. Let $m: X \times X \rightarrow X$ denote the group law, and consider

$$
m^{-1}(C) \cap(C \times X)
$$

as a family over $C$ via first projection. The fibre over $p \in C$ is $C_{p}$. Let $N_{d}=V\left(\mathfrak{m}_{0}^{d}\right)$ be the $d-1$ 'st order infinitesimal neighbourhood of the origin in $X$. Then

$$
Z=m^{-1}(C) \cap\left(C \times N_{d}\right) \subset C \times X
$$

is a $C$-flat family of degree $d$ subschemes in $X$; its fibre over $p \in C$ is $C_{p} \cap N_{d}$. This family defines $\phi_{+}: C \rightarrow \operatorname{Hilb}^{d}(X)$, and we let $\phi_{-}=-\phi_{+}$(where the minus sign denotes the automorphism of $\operatorname{Hilb}^{d}(X)$ induced by the group inverse in $\left.X\right)$.

Lemma 2.2. The map $\phi_{+}: C \rightarrow \operatorname{Hilb}^{d}(X)$ is a closed embedding for $d>2$.

In the proof of the Lemma, we shall make use of the difference map $\delta: C \times C \rightarrow X$, sending a pair $(p, q)$ to the degree zero divisor $p-q$. We let $C-C \subset X$ denote its image. If $C$ is hyperelliptic, we may and will choose the Abel-Jacobi embedding $C \subset X$ such that the involution -1 on $X$ restricts to the hyperelliptic involution $\iota$ on $C$. Thus, when $C$ is hyperelliptic, $C-C$ coincides with the distinguished surface $W_{2}$, and the difference map $\delta$ can be factored via the symmetric product $C^{(2)}$ :

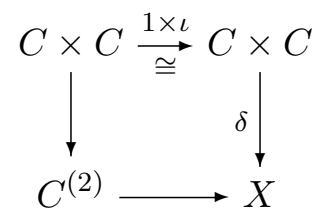

We note that the double cover $C \times C \rightarrow C^{(2)}$, that sends an ordered pair to the corresponding unordered pair, is branched along the diagonal, so that via $1 \times \iota$, the branching divisor becomes the "antidiagonal" $(1, \iota): C \hookrightarrow C \times C$.

As is well known, the surface $C-C$ is singular at 0 , and nonsingular everywhere else. The blowup of $C-C$ at 0 coincides with $\delta: C \times C \rightarrow C-C$ when $C$ is nonhyperelliptic, and with the addition map $C^{(2)} \rightarrow W_{2}$ when $C$ is hyperelliptic.

Proof of Lemma 2.2. To prove that $\phi_{+}$is a closed embedding, we need to show that its restriction to any finite subscheme $T \subset C$ of degree 2 is nonconstant, i.e. that the family $\left.Z\right|_{T}$ is not a product $T \times \Gamma$. For this it suffices to prove that if $\Gamma$ is a finite scheme such that

$$
m^{-1}(C) \supset T \times \Gamma
$$

then the degree of $\Gamma$ is at most 2 . 
Consider the following commutative diagram:

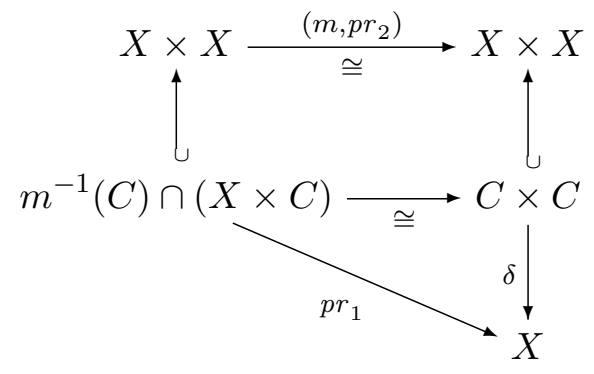

First suppose $T=\{p, q\}$ with $p \neq q$. The claim is then simply that $C_{p} \cap C_{q}$, or equivalently its translate $C \cap C_{q-p}$, is at most a finite scheme of degree 2. Diagram (2) identifies the fibre $\delta^{-1}(q-p)$ on the right with precisely $C \cap C_{q-p}$ on the left. But $\delta^{-1}(q-p)$ is a point when $C$ is nonhyperelliptic, and two points if $C$ is hyperelliptic.

Next suppose $T \subset C$ is a nonreduced degree 2 subscheme supported in $p$. Assuming $\Gamma$ satisfies (1), we have $\Gamma \subset C_{p}$, so

$$
m^{-1}(C) \cap\left(X \times C_{p}\right) \supset T \times \Gamma
$$

or equivalently

$$
m^{-1}(C) \cap(X \times C) \supset T_{p} \times \Gamma_{-p}
$$

We have $T_{p} \subset C-C$, and Diagram (2) identifies $\delta^{-1}\left(T_{p}\right)$ on the right with $m^{-1}(C) \cap\left(T_{p} \times C\right)$ on the left.

Suppose $C$ is nonhyperelliptic. Then $\delta$ is the blowup of $0 \in C-C$, and $\delta^{-1}\left(T_{p}\right)$ is the diagonal $\Delta_{C} \subset C \times C$ together with an embedded point of multiplicity 1 (corresponding to the tangent direction of $T_{p} \subset C-C$ ). Diagram (2) identifies the diagonal in $C \times C$ on the right with $\{0\} \times C$ on the left. Thus $m^{-1}(C) \cap\left(T_{p} \times C\right)$ is $\{0\} \times C \subset X \times C$ with an embedded point. Equivalently, $m^{-1}(C) \cap\left(T \times C_{p}\right)$ is $\{p\} \times C_{p}$ with an embedded point, say at $(p, q)$. This contains no constant family $T \times \Gamma$ except for $\Gamma=\{q\}$, so $\Gamma$ has at most degree 1 .

Next suppose $C$ is hyperelliptic. We claim that $\delta^{-1}\left(T_{p}\right)$ is the diagonal $\Delta_{C} \subset C \times C$ with either two embedded points of multiplicity 1 , or one embedded point of multiplicity 2 . As in the previous case, this implies that $m^{-1}(C) \cap\left(T \times C_{p}\right)$ is $\{p\} \times C_{p}$ with two embedded points of multiplicity 1 or one embedded point of multiplicity 2 , and the maximal constant family $T \times \Gamma$ it contains has $\Gamma$ of degree 2 . It remains to prove that $\delta^{-1}\left(T_{p}\right)$ is as claimed.

We have $W_{2}=C-C$, and the blowup at 0 is $C^{(2)} \rightarrow W_{2}=C-C$. The preimage of $T_{p}$ is the curve $(1+\iota): C \rightarrow C^{(2)}$, together with an embedded point of multiplicity 1 , say supported at $q+\iota(q)$. Now the two to one cover $C \times C \rightarrow C^{(2)}$ is branched along the diagonal $2 C \subset C^{(2)}$, If $q \neq \iota(q)$, then the preimage in $C \times C$ is just $(1, \iota): C \rightarrow C \times C$, together with two embedded points of multiplicity 1 , supported at $(q, \iota(q))$ and $(\iota(q), q)$. If $q=\iota(q)$, i.e. $q$ is Weierstraß, then we claim the preimage in $C \times C$ is $(1, \iota): C \rightarrow C \times C$ together with an embedded point of multiplicity 2 . This follows once we know that the curves $2 C$ and $(1+\iota)(C)$ in $C^{(2)}$ intersect 
transversally. And they do, as the tangent spaces of the two curves $(1,1)(C)$ (the diagonal) and $(1, \iota)(C)$ in $C \times C$ are invariant under the involution exchanging the two factors, with eigenvalues 1 and -1 , respectively.

Proof of Proposition 2.1. Point (1) is obvious, so we may assume $X=$ Jac $C$. By Lemma 2.2, $\phi_{+}$is a closed embedding and hence so is $\phi_{-}=-\phi_{+}$. If $C$ is hyperelliptic, we have chosen the embedding $C \subset X$ such that the involution -1 on $X$ extends the hyperelliptic involusion $\iota$ on $C$. It follows that $C_{p}=-C_{\iota(p)}$, and thus $\phi_{-}=\phi_{+} \circ \iota$. Thus the two maps $\phi_{+}$and $\phi_{-}$have coinciding image, and (2) follows.

For (3), it remains to prove that if $C$ is nonhyperelliptic, then the images of $\phi_{-}$and $\phi_{+}$are disjoint, i.e. we never have $C_{p} \cap N_{d}=\left(-C_{q}\right) \cap N_{d}$ for distinct points $p, q \in C$. In fact, $C_{p} \cap\left(-C_{q}\right)$ is at most a finite scheme of degree 2 : the addition map

$$
C \times C \rightarrow X
$$

is a degree two branched cover of $C^{(2)} \cong W_{2}$ (using that $C$ is nonhyperelliptic), and its fibre over $p+q \in W_{2}$ is isomorphic to $C_{p} \cap\left(-C_{q}\right)$.

\section{Proof of Theorem A}

In view of Proposition 2.1, it suffices to prove that $Y$ in Theorem A agrees with $Y_{3}$ in Proposition 2.1. This is a reformulation of the Gunning-Welters criterion: given $\Gamma \in \operatorname{Hilb}^{3}(X)$, consider the set

$$
V_{\Gamma}=\left\{x \in X \mid \Gamma_{X} \subset \psi^{-1}(\ell) \text { for some line } \ell \subset \mathbb{P}^{2^{g}-1}\right\} .
$$

Then Gunning-Welters says that $V_{\Gamma}$ has positive dimension if and only if $(X, \Theta)$ is a Jacobian. Moreover, when $V_{\Gamma}$ has positive dimension, it is a smooth curve, the canonical map $\operatorname{Jac}\left(V_{\Gamma}\right) \rightarrow X$ is an isomorphism, and $\Gamma$ is contained in $V_{\Gamma}$ (see [15, Theorem (0.4)]). Thus $Y$ in Theorem A agrees with $Y_{3}$ in Proposition 2.1.

\section{Proof of Theorem B}

Let $X$ be the Jacobian of $C$. For convencience, we fix an Abel-Jacobi embedding $C \hookrightarrow X$. First, we shall analyse theta-genericity for finite subschemes of $C$.

Recall the notion of theta-duality: whenever $V \subset X$ is a closed subscheme, we let

$$
T(V)=\left\{x \in X \mid V \subset \Theta_{x}\right\} .
$$

It has a natural structure as a closed subscheme of $X$ (see [13, Section 4] and [6, Section 2.2]); the definition as a (closed) subset is sufficient for our present purpose.

With this notation, theta-genericity means that for all chains of subschemes

$$
\Gamma_{1} \subset \Gamma_{2} \subset \cdots \subset \Gamma_{g+1} \subset \Gamma,
$$


where $\Gamma_{i}$ has degree $i$, the corresponding chain of theta-duals,

$$
T\left(\Gamma_{1}\right) \supset T\left(\Gamma_{2}\right) \supset \cdots \supset T\left(\Gamma_{g+1}\right),
$$

consists of strict inclusions of sets.

We write $\widehat{\mathscr{F}}$ for the Fourier-Mukai transform [10, 8] of a WIT-sheaf $\mathscr{F}$ on $X$ [10, Def. 2.3]: $\widehat{\mathscr{F}}$ is a sheaf on the dual abelian variety, which we will identify with $X$ using the principal polarization.

Proposition 4.1. Let $\Gamma \subset C$ be a finite subscheme of degree at least $g+1$. Then $\Gamma$ is thetageneral, as a subscheme of $\operatorname{Jac}(C)$, if and only if $\operatorname{dim} H^{0}\left(\mathscr{O}_{C}\left(\Gamma_{g}\right)\right)=1$ for every degree $g$ subscheme $\Gamma_{g} \subset \Gamma$. In particular, if $\Gamma$ is supported at a single point $p \in C$, then $\Gamma$ is theta-general if and only if $p$ is not a Weierstraß point.

Proof. For the last claim, note that the condition $\operatorname{dim} H^{0}\left(\mathscr{O}_{C}(g p)\right)>1$ says precisely that $p$ is a Weierstraß point.

For any effective divisor $\Gamma_{g} \subset C$ degree $g$, it is well known that $\operatorname{dim} H^{0}\left(\mathscr{O}_{C}\left(\Gamma_{g}\right)\right)=1$ if and only if $\Gamma_{g}$ can be written as the intersection of $C \subset \operatorname{Jac}(C)$ and a $\Theta$-translate (this is one formulation of Jacobi inversion). If this is the case, then the point $x \in X$ satisfying $\Gamma_{g}=C \cap \Theta_{x}$ is unique.

Consider a chain (3). If there is a degree $g$ subscheme $\Gamma_{g} \subset \Gamma$ not of the form $C \cap \Theta_{x}$, then every $\Theta$-translate containing $\Gamma_{g}$ also contains $C$, and in particular $T\left(\Gamma_{g}\right)=T\left(\Gamma_{g+1}\right)$. Hence $\Gamma$ is not theta-general.

Suppose, on the other hand, that $\Gamma_{g}$ is of the form $C \cap \Theta_{x}$. Then $T\left(\Gamma_{g}\right) \backslash T\left(\Gamma_{g+1}\right)$ consists (as a set) of exactly the point $x$. Thus there is a Zariski open neighbourhood $U \subset X$ of $x$ such that $T\left(\Gamma_{g}\right) \cap U=\{x\}$. We claim that, for a possibly smaller neighbourhood $U$, there are regular functions $f_{1}, \ldots, f_{g} \in \mathscr{O}_{X}(U)$, such that $T\left(\Gamma_{i}\right) \cap U=V\left(f_{1}, \ldots, f_{i}\right)$ for all $i$ : in fact, apply the Fourier-Mukai functor to the short exact sequence

$$
0 \rightarrow \mathscr{I}_{\Gamma_{i}}(\Theta) \rightarrow \mathscr{O}_{X}(\Theta) \rightarrow \mathscr{O}_{\Gamma_{i}} \rightarrow 0
$$

to obtain

$$
0 \rightarrow \mathscr{O}_{X}(-\Theta) \stackrel{F_{i}}{\rightarrow} \widehat{\widehat{\mathscr{O}}_{\Gamma_{i}}} \rightarrow \widehat{\mathscr{I}_{\Gamma_{i}}(\Theta)} \rightarrow 0
$$

Then $F_{i}$ is a section of a locally free sheaf of rank $i$, and its vanishing locus is exactly $T\left(\Gamma_{i}\right)$. Choose trivializations of $\widehat{\mathscr{O}_{\Gamma_{i}}}$ over $U$ for all $i$ compatibly, in the sense that the surjections $\widehat{\mathscr{O}_{\Gamma_{i+1}}} \rightarrow \widehat{\mathscr{O}_{\Gamma_{i}}}$ correspond to projection to the first $i$ factors. Then $F_{i}=\left(f_{1}, \ldots, f_{i}\right)$ in these trivializations.

As $T\left(\Gamma_{g}\right) \cap U$ is zero dimensional, it follows that each $T\left(\Gamma_{i}\right) \cap U$ has codimension $i$ in $U$. Hence all the inclusions $T\left(\Gamma_{i}\right) \supset T\left(\Gamma_{i+1}\right)$ are strict, and so $\Gamma$ is theta-general.

Now we can compare the locus $Y$ in Theorem B with $Y_{g+2}$ in Proposition 2.1 by means of the Castelnuovo-Schottky theorem: 
Corollary 4.2 (of Theorem 1.3). Let $\Gamma \in \operatorname{Hilb}^{g+2}(X)$ be theta-general and supported at $0 \in X$. Then $\Gamma$ is in the locus $Y_{g+2}$ in Propsition 2.1 if and only if it is in special position with respect to $2 \Theta$-translates.

Proof. Theorem 1.3 immediately shows that if $\Gamma$ is in special position with respect to $2 \Theta-$ translates, then $\Gamma \in Y_{g+2}$.

The converse is straight forward, and does not require the theta-genericity assumption. Indeed, we use that any curve $C^{\prime} \subset X$ for which $\operatorname{Jac}\left(C^{\prime}\right) \rightarrow X$ is an isomorphism is of the form $\pm C_{p}$ for some $p \in X$ and we claim that if $\Gamma \subset \pm C_{p}$, then $\Gamma$ is in special position with respect to $2 \Theta$-translates. For ease of notation, we rename $\pm C_{p}$ as $C$, so that $\Gamma \subset C$. Then $H^{0}\left(\mathscr{I}_{C}\left(2 \Theta_{x}\right)\right) \subset H^{0}\left(\mathscr{I}_{\Gamma}\left(2 \Theta_{x}\right)\right)$, and the exact sequence

$$
0 \rightarrow H^{0}\left(\mathscr{I}_{C}\left(2 \Theta_{x}\right)\right) \rightarrow H^{0}\left(\mathscr{O}_{X}\left(2 \Theta_{x}\right)\right) \rightarrow H^{0}\left(\mathscr{O}_{C}\left(2 \Theta_{x}\right)\right)
$$

shows that already the codimension of $H^{0}\left(\mathscr{I}_{C}\left(2 \Theta_{x}\right)\right)$ in $H^{0}\left(\mathscr{O}_{X}\left(2 \Theta_{x}\right)\right)$ is at most $\operatorname{dim} H^{0}\left(\mathscr{O}_{C}\left(2 \Theta_{x}\right)\right)=$ $g+1$.

Theorem B now follows: The set $Y$ defined there agrees with the theta-general elements in $Y_{g+2}$, by the Corollary. By Proposition 4.1, $\Gamma=\phi_{ \pm}(p)$ is theta-general if and only if the supporting point 0 of $\Gamma$ is not Weierstraß in $\pm C_{p}$, i.e. $p \in C$ is not Weierstraß.

\section{Historical REMARK}

Assume $C$ is not hyperelliptic. Then $C_{p} \cap\left(-C_{p}\right)$ is a finite subscheme of degree 2 supported at 0 . Thus, for $d=2$, we have $\phi_{+}=\phi_{-}$, and the argument in Lemma 2.2 shows that $\phi_{+}$is an isomorphism from $C$ onto $Y_{2}$. If $C$ is hyperelliptic with hyperelliptic involution $\iota$, however, we find that $\phi_{+}$factors through $C / \iota \cong \mathbb{P}^{1}$, and $Y_{2} \cong \mathbb{P}^{1}$, and we cannot reconstruct $C$ from $Y_{2}$ alone.

In the nonhyperelliptic situation, it is well known that the curve $C$ can be reconstructed as the projectivized tangent cone to the surface $C-C \subset X$ at 0 . This projectivized tangent cone is exactly $Y_{2}$ (when we identify the projectivized tangent space to $X$ at 0 with the closed subset of $\operatorname{Hilb}^{2}(X)$ consisting of nonreduced degree 2 subschemes supported at 0 ). To quote Mumford [11]: "If $C$ is hyperelliptic, other arguments are needed." In the present note, these other arguments are to increase $d$ !

Acknowledgements. The authors were partially funded by the NILS project UCM-EEAABEL-03-2010 to visit respectively the University of Barcelona and the Stord/Haugesund University College. Moreover, parts of this project were written while the authors were visiting the University of Bonn. The warm hospitality from these institutions is gratefully acknowledged. 


\section{REFERENCES}

[1] E. Arbarello. Survey of work on the Schottky problem up to 1996. In The red book of varieties and schemes, volume 1358 of Lecture Notes in Mathematics, pages 287-291. Springer-Verlag, Berlin, 1999.

[2] A. Beauville. Le problème de Schottky et la conjecture de Novikov. Astérisque, 152-153:101-112, 1987. Exp. 675 du Séminaire Bourbaki.

[3] O. Debarre. The Schottky problem: an update. In Current topics in complex algebraic geometry (Berkeley, CA, 1992/93), volume 28 of Math. Sci. Res. Inst. Publ., pages 57-64. Cambridge Univ. Press, Cambridge, 1995.

[4] S. Grushevsky. Cubic equations for the hyperelliptic locus. Asian J. Math., 8(1):161-172, 2004.

[5] S. Grushevsky. Erratum to "Cubic equations for the hyperelliptic locus". Asian J. Math., 9(2):273-274, 2005.

[6] M. G. Gulbrandsen and M. Lahoz. Finite subschemes of abelian varieties and the Schottky problem. Ann. Inst. Fourier (Grenoble), 61(5):2039-2064, 2011.

[7] R. C. Gunning. Some curves in abelian varieties. Invent. Math., 66(3):377-389, 1982.

[8] D. Huybrechts. Fourier-Mukai transforms in algebraic geometry. Oxford Mathematical Monographs. The Clarendon Press Oxford University Press, Oxford, 2006.

[9] I. Krichever. Characterizing Jacobians via trisecants of the Kummer variety. Ann. Math. (2), 172(1):485-516, 2010.

[10] S. Mukai. Duality between $D(X)$ and $D(\hat{X})$ with its application to Picard sheaves. Nagoya Math. J., 81:153$175,1981$.

[11] D. Mumford. Curves and their Jacobians. The University of Michigan Press, Ann Arbor, Mich., 1975.

[12] G. Pareschi and M. Popa. Castelnuovo theory and the geometric Schottky problem. J. Reine Angew. Math., 615:25-44, 2008.

[13] G. Pareschi and M. Popa. Generic vanishing and minimal cohomology classes on abelian varieties. Math. Ann., 340(1):209-222, 2008.

[14] G. E. Welters. On flexes of the Kummer variety (note on a theorem of R. C. Gunning). Nederl. Akad. Wetensch. Indag. Math., 45(4):501-520, 1983.

[15] G. E. Welters. A criterion for Jacobi varieties. Ann. of Math. (2), 120(3):497-504, 1984.

University of Stavanger, Department of Mathematics and Natural Sciences, NO-4036 StaVANGER, NORWAY

E-mail address: martin.gulbrandsen@uis.no

Institut de Mathématiques Jussieu - Paris Rive Gauche. Université Paris Diderot - Paris 7.

Bâtiment Sophie-Germain. Case 7012. F-75205 Paris Cedex 13, France.

E-mail address: marti.lahoz@imj-prg.fr 